\title{
PROOF OF A THEOREM OF THORIN
}

Let

\author{
J. D. TAMARKIN AND A. ZYGMUND
}

$$
A(x, y)=\sum_{j, k=1}^{m, n} a_{j k} x_{j} y_{k}
$$

be a complex bilinear form, and let $M(\alpha, \beta)$ denote the upper bound of $|A(x, y)|$ for the $x$ 's and $y$ 's satisfying the condition

$$
\left(\sum\left|x_{i}\right|^{1 / \alpha}\right)^{\alpha} \leqq 1, \quad\left(\sum\left|y_{k}\right|^{1 / \beta}\right)^{\beta} \leqq 1 .
$$

Then $\log M(\alpha, \beta)$ is a convex function of the point $(\alpha, \beta)$ in the triangle

$$
0 \leqq \alpha \leqq 1, \quad 0 \leqq \beta \leqq 1, \quad \alpha+\beta \geqq 1 .
$$

The above result is due to $M$. Riesz. ${ }^{1}$ In a recent paper it was shown by Thorin ${ }^{2}$ that the theorem is restricted neither to bilinear forms nor to the triangle (1). Thorin's result is as follows.

THEOREM. (i) Let $f\left(z_{1}, z_{2}, \cdots, z_{r}\right)$ be an entire function of $r$ complex variables $z_{1}, z_{2}, \cdots, z_{r}$. Let $K$ be a bounded domain $\left(v_{1}, v_{2}, \cdots, v_{r}\right)$ of the $r$-dimensional Euclidean space, satisfying the conditions $v_{1} \geqq 0$, $v_{2} \geqq 0, \cdots, v_{r} \geqq 0$. Let $M\left(\alpha_{1}, \alpha_{2}, \cdots, \alpha_{r}\right)$ denote the upper bound of $\left|f\left(z_{1}, z_{2}, \cdots, z_{r}\right)\right|$ for

$$
\left|z_{1}\right|=v_{1}^{\alpha_{1}}, \cdots,\left|z_{r}\right|=v_{r}^{\alpha_{r}}, \quad \text { and }\left(v_{1}, v_{2}, \cdots, v_{r}\right) \in K .
$$

Then $\log M\left(\alpha_{1}, \alpha_{2}, \cdots, \alpha_{r}\right)$ is a convex function of the point $\left(\alpha_{1}, \alpha_{2}, \cdots, \alpha_{r}\right)$ in the domain $0 \leqq \alpha_{j}<+\infty, j=1,2, \cdots, r$.

(ii) If the points of $K$ satisfy a condition

$$
0<A \leqq v_{j} \leqq B<+\infty \quad(j=1,2, \cdots, r)
$$

then $\log M\left(\alpha_{1}, \alpha_{2}, \cdots, \alpha_{r}\right)$ is convex in the whole space $-\infty<\alpha_{j}<+\infty$, $j=1,2, \cdots, r$.

In case (ii), vanishing of some of the $\alpha$ 's does not require additional discussion. The situation is slightly different in case (i), since $v_{j}^{\alpha_{i}}$ has no meaning if both $v_{j}$ and $\alpha_{j}$ are zero. The sets $z_{1}, z_{2}, \cdots, z_{r}$

Received by the editors January 6, 1944.

$1 \mathrm{M}$. Riesz, Sur les maxima des formes bilinéaires et sur les fonctionnelles lineaires, Acta Math. vol. 49 (1926) pp. 465-497.

${ }^{2} \mathrm{G}$. O. Thorin, An extension of a convexity theorem due to $M$. Riesz, Kungl. Fysiografiska Sällskapets i Lund Förhandlinger vol. 8 (1939) no. 14. 
for which this occurs have simply to be disregarded in the definition of $M\left(\alpha_{1}, \alpha_{2}, \cdots, \alpha_{r}\right)$.

Owing to the importance of the theorem (even in the special case of M. Riesz), any simplification of its proof may be of interest. The proof given below seems to be slightly simpler than the original one of Thorin, and is based on the following elementary principle. ${ }^{3}$

(a) A necessary and sufficient condition that a real valued function $\phi(t)$ be convex in an interval $(a, b)$ is that, for any subinterval $\left(a^{\prime}, b^{\prime}\right)$ and any real number $\mu$, the maximum of the function $\phi(t)+\mu t$ in $\left(a^{\prime}, b^{\prime}\right)$ be attained at least at one of the end points $a^{\prime}, b^{\prime}$.

Its proof is immediate. If $\phi(t)$ is convex, so is $\phi_{1}(t)=\phi(t)+\mu t$. Since no point of the arc $y=\phi_{1}(t), a^{\prime} \leqq t \leqq b^{\prime}$, lies above the chord joining its end points, the maximum of $\phi_{1}(t)$ in $\left(a^{\prime}, b^{\prime}\right)$ is either at $t=a^{\prime}$ or $t=b^{\prime}$. Conversely, if $\phi(t)$ is not convex in $(a, b)$, there is an arc $y=\phi(t)$, $a^{\prime} \leqq t \leqq b^{\prime}$, which lies partly above the corresponding chord. If $y=-\mu t-\nu$ is the equation of the chord, the sum $\phi(t)+\mu t+\nu$ vanishes for $t=t_{1}, t_{2}$, and takes on positive values at some points inside $\left(a^{\prime}, b^{\prime}\right)$. Hence $\phi(t)+\mu t$ does not attain its maximum in $\left(a^{\prime}, b^{\prime}\right)$ at either of the end points $a^{\prime}, b^{\prime}$.

The following form of the principle will be more convenient for our purposes.

(b) A necessary and sufficient condition that the logarithm of a nonnegative function $\phi(t)$ be convex in an interval $(a, b)$ is that, for any subinterval $\left(a^{\prime}, b^{\prime}\right)$ and any real $\mu$, the maximum of the function $\phi(t) e^{\mu t}$ in $\left(a^{\prime}, b^{\prime}\right)$ be attained at least at one of the end points $a^{\prime}, b^{\prime}$.

The necessity of the condition follows from (a). So does the sufficiency; if $\phi(t)$ is strictly positive. It is, however, easy to show that if $\phi(t)$ is non-negative, satisfies the condition of $(b)$, and vanishes at a point $t_{0}$ of $(a, b)$, then $\phi(t)$ vanishes identically inside $(a, b)$. For if $\phi(t)$ were positive at a point $t_{1}$ which lies, for example, to the right of $t_{0}$, so that $t_{0}<t_{1}<b$, and if $t_{1}<t_{2}<b$, then $\phi(t) e^{\mu\left(t-t_{1}\right)}$ is 0 at $t_{0}$, is $\phi\left(t_{1}\right)$ at $t_{1}$, and is very small at $t_{2}$, if $\mu$ is negative and large enough. Hence the maximum of $\phi(t) e^{\mu\left(t-t_{1}\right)}$ in $\left(t_{0}, t_{2}\right)$ is attained at neither end point. This proves (b), if we agree to consider the function which is $-\infty$ everywhere inside $(a, b)$ as convex.

3 This principle, both in forms (a) and (b), was first stated by S. Saks and used by him to simplify certain proofs from the theory of functions. See his papers Sur un theoreme de M. Montel, C. R. Acad. Sci. Paris vol. 187 (1928) pp. 276-277, and On the properties of convex and subharmonic functions (in Polish), Mathesis Polska vol. 6 (1930). 
We now pass to the proof of the theorem of Thorin, starting with part (ii). Let

$$
v_{i}=e^{k_{i}}, \quad j=1,2, \cdots, r .
$$

If $\left(v_{1}, v_{2}, \cdots, v_{r}\right) \in K$, the $\xi$ 's are bounded. Obviously, we may assume here that $K$ is closed. Hence

$$
M\left(\alpha_{1}, \alpha_{2}, \cdots, \alpha_{r}\right)=\max \left|f\left(e^{\alpha_{1} \xi_{1}+i_{1}}, \cdots, e^{\alpha_{r} \xi_{r}+i_{r}}\right)\right|
$$

for $\left(e^{\xi_{1}}, e^{\xi_{2}}, \cdots, e^{\xi_{r}}\right) \in K$ and $\eta_{1}, \eta_{2}, \cdots, \eta_{r}$ arbitrary. Since $\left|f\left(e^{\alpha_{1} \xi_{1}+i \eta 1}, \cdots\right)\right|$ is a continuous function of the variables $\alpha_{j}, \xi_{j}, \eta_{j}$, it follows that $M\left(\alpha_{1}, \alpha_{2}, \cdots, \alpha_{r}\right)$ is a continuous function of the $\alpha$ 's. In order to show that its logarithm is convex on any straight line

$$
\alpha_{1}=\alpha_{1}^{0}+\lambda_{1} t, \alpha_{2}=\alpha_{2}^{0}+\lambda_{2} t, \cdots, \alpha_{r}=\alpha_{r}^{0}+\lambda_{r} t,
$$

it is enough to show that, for any real $\mu$, the product

$$
M\left(\alpha_{1}^{0}+\lambda_{1} t, \cdots, \alpha_{r}^{0}+\lambda_{r} t\right) e^{\mu t}
$$

has no proper maximum for any finite $t$.

Let us assume, contrary to this, that for some $\mu$ such a maximum does exist. By a change of variable we may adjust it so that the maximum is attained for $t=0$. Then

$$
M\left(\alpha_{1}^{0}, \alpha_{2}^{0}, \cdots, \alpha_{r}^{0}\right) \geqq M\left(\alpha_{1}^{0}+\lambda_{1} t, \alpha_{2}^{0}+\lambda_{2} t, \cdots, \alpha_{r}^{0}+\lambda_{r} t\right) e^{\mu t}
$$

for small $|t|$, and we have strict inequality for some $t$ 's arbitrarily small. We may write

$$
M\left(\alpha_{1}^{0}, \cdots, \alpha_{r}^{0}\right)=\left|f\left(e^{\alpha_{1}^{0} \xi_{1}^{0}+i \eta_{1}^{0}}, \cdots, e^{\alpha_{r}^{0} \xi_{r}^{0}+i \eta_{r}^{0}}\right)\right|,
$$

where $\left(e^{\xi_{1}^{0}}, e_{\xi_{1}^{0}}^{\eta_{1}}, \cdots, e_{r}^{\xi_{r}^{0}}\right)$ is a point of $K$, and the $\eta_{1}^{0}, \eta_{2}^{0}, \cdots, \eta_{r}^{0}$ are suitably chosen. Thus

$$
\begin{aligned}
&\left|f\left(e^{\alpha_{1}^{0} \xi_{1}^{0}+i \eta_{1}^{0}}, \cdots, e^{\alpha_{r}^{0} \xi_{r}^{0}+i \eta_{r}^{0}}\right)\right| \\
& \geqq\left|f\left(e^{\left(\alpha_{1}^{0}+\lambda_{1} t\right) \xi_{1}^{0}+i_{1}}, \cdots, e^{\left(\alpha_{r}^{0}+\lambda_{r} t\right) \xi_{r}^{0}+i \eta_{r}}\right) e^{\mu t}\right|
\end{aligned}
$$

for all real $t$ 's sufficiently small in absolute value, and for all real $\eta$ 's. We assert that (3) holds for all complex $t$ 's of sufficiently small modulus. This follows immediately, for if we replace $t$ by $t+i v$ in (3), the only effect will be to change the $\eta$ 's. It follows in particular that the modulus of the entire function (in $t$ )

$$
f\left(e^{\left(\alpha_{1}^{0}+\lambda_{1} t\right) \xi_{1}^{0}+i \eta_{1}^{0}}, \cdots, e^{\left.\left(\alpha_{r}^{0}+\lambda_{r} t\right) \xi_{r}^{0}+i \eta_{r}^{0}\right)} e^{\mu t}\right.
$$

attains a proper maximum at $t=0$, which contradicts the principle 
of the maximum for analytic functions. Hence part (ii) of the theorem is proved.

As Thorin himself points out, part (i) of the theorem is an immediate consequence of part (ii). For if $K_{\mathrm{e}}$ is the part of $K$ for which $v_{j} \geqq \epsilon, j=1,2, \cdots, r$, and if $M_{\epsilon}\left(\alpha_{1}, \alpha_{2}, \cdots, \alpha_{r}\right)$ is the function $M\left(\alpha_{1}, \alpha_{2}, \cdots, \alpha_{r}\right)$ corresponding to $K_{\epsilon}$, then

$$
\lim _{\epsilon \rightarrow 0} M_{\epsilon}\left(\alpha_{1}, \alpha_{2}, \cdots, \alpha_{r}\right)=M\left(\alpha_{1}, \alpha_{2}, \cdots, \alpha_{r}\right) .
$$

This holds both when all the $\alpha$ 's are different from zero and when some of them vanish. Hence $M\left(\alpha_{1}, \alpha_{2}, \cdots, \alpha_{r}\right)$ is convex for $\alpha_{1} \geqq 0, \alpha_{2} \geqq 0, \cdots, \alpha_{r} \geqq 0$. The restriction $\alpha_{j} \geqq 0$ is introduced to avoid infinite values for $M$.

Let $k>0$. The theorem of Thorin may be considered as the limiting case $(k=+\infty)$ of a similar theorem in which the expression $M\left(\alpha_{1}, \alpha_{2}, \cdots, \alpha_{r}\right)$ is replaced by

$$
\begin{aligned}
M_{k}\left(\alpha_{1}, \alpha_{2}, \cdots, \alpha_{r}\right) & \\
& =\max \int_{0}^{2 \pi} \cdots \int_{0}^{2 \pi}\left|f\left(e^{\alpha_{1} \xi_{1}+i \eta_{1}}, \cdots, e^{\alpha_{r} \xi_{r}+i \eta_{r}}\right)\right|^{k} d \eta_{1} \cdots d \eta_{r}
\end{aligned}
$$

and $\left(e^{\xi_{1}}, \cdots, e^{\xi_{r}}\right) \in K$. The limitations on the $\alpha^{\prime}$ s are the same as before. The proof does not differ from the one given above, if we use the fact that the integral

$$
\int_{0}^{2 \pi} \cdots \int_{0}^{2 \pi} \mid f\left(e^{\left(\alpha_{1}^{0}+\lambda_{1} t\right) \xi_{1}^{0}+i \eta_{1}}, \cdots, e^{\left(\alpha_{r}^{0}+\lambda_{r} t\right) \xi_{r}^{0}+i \eta_{r}} \mid k d \eta_{1} \cdots d \eta_{r}\right.
$$

is a subharmonic function of the (complex) variable $t$, and so cannot attain a proper maximum. That this integral is a subharmonic function of $t$ follows from the fact that $\left|f\left(e^{\left(\alpha_{1}^{0}+\lambda_{1} t\right) \xi_{1}^{0}+i \eta 1}, \cdots\right)\right|^{k}$ is subharmonic in $t$ for every fixed set of $\eta_{1}, \eta_{2}, \cdots, \eta_{r}$.

As Thorin pointed out, the function $f\left(z_{1}, z_{2}, \cdots, z_{r}\right)$ of his theorem need not be entire. If it is regular in a domain $D$, the function $M\left(\alpha_{1}, \cdots, \alpha_{r}\right)$ is convex in a domain of the variables $\alpha_{1}, \cdots, \alpha_{r}$. The same remark applies to $M_{k}$.

BROWN UNIVERSITY AND Mount Holyoke College 